\title{
3rd Generation accessibility: information and communication technologies towards universal access
}

\author{
Maria Fernanda Cabrera-Umpiérrez
}

Published online: 13 August 2014

(c) Springer-Verlag Berlin Heidelberg 2014

New technologies offer several opportunities for people with disabilities, such as the acquisition of new skills in using innovative assistive technologies, the raise of their employability and the increase in their levels of social integration. The impact that accessible information and communication technologies (ICT) could have on the lives of people with disabilities is mainly centred on facilitating their everyday activities and job opportunities (in the mainstream labour market) and providing support on a daily basis, whether it is to access their phone, mobile or computer or even the TV. However, while for most of the existing devices an accessible choice is available, accessibility was never the main focus during the development phase of those products. Their accessibility features are more an add-on than a really embedded feature. Clearly, there is a need to make assistive technologies widely available to a large group of citizens, but the fact is that there is no significant generalised accessibility support. The 3rd generation of accessible ICT applications, tools and systems constitutes a main bridge for facilitating universal access to ICT through embedding accessibility support in the core of web, desktop and mobile environments. The exploitation of these technologies, behind the recent expansion of accessibility tools and standardisation methodologies, provides a new approach for generalised access to mainstream ICT and contributes towards better accessibility for all citizens in all context of use, empowering individuals to fully participate in the knowledge society.

This special issue comes after the successful finalisation of two European research projects: AEGIS (Open

M. F. Cabrera-Umpiérrez $(\bowtie)$

Life Supporting Technologies, Universidad Politecnica de Madrid, Madrid, Spain

e-mail: chiqui@1st.tfo.upm.es
Accessibility Everywhere: Groundwork, Infrastructure, Standards; http://www.aegis-project.eu), and ACCESSIBLE (Accessibility Assessment Simulation Environment for New Applications Design and Development; http:// www.accessible-eu.org/), both partially funded under the EU 7th Framework Programme. This UAIS special issue is focused on research work related to the design, development, evaluation and use of new ICT applications, tools and systems that integrate support for accessibility into every aspect of ICT in order to enhance the quality of life and personal autonomy of all citizens. As a result, seven (7) papers describing methodologies, systems, case studies and evaluations from the universal access area comprise this special issue.

Heiko Desruelle, Simon Isenberg, Andreas Botsikas, Paolo Vergori and Frank Gielen's “Accessible user interface support for multi-device ubiquitous applications. Architectural modifiability considerations" presents the core architectural modifiability considerations for designing a multidevice ubiquitous platform for accessible web-based applications using the Webinos platform. Five proof-of-concept application scenarios were selected to demonstrate the potential impact. The five applications were evaluated in different focus groups using Likert questions. The input of 41 participants from across Europe was collected, confirming people's need for accessible human computer interaction within the ubiquitous computing domain.

In their paper, "Towards Standardization of User Models for Simulation and Adaptation Purposes", Kaklanis $\mathrm{N}$. et al., present the efforts of the VUMS cluster of projects towards the development of an interoperable user model, able to describe both able-bodied and people with various kinds of disabilities. The results show the definition of VUMS Exchange Format, a common format for the definition of user models. 
Vassilis Koutkias, Nick Kaklanis, Konstantinos Votis, Dimitrios Tzovaras and Nicos Maglaveras, in their paper "An Integrated Semantic Framework Supporting Universal Accessibility to ICT", present an integrated ontological framework for the semantic representation of terms and concepts that are required for addressing the universal accessibility in the scope of the Cloud4all project and the Global Public Inclusive Infrastructure (GPII). The proposed framework aspires to facilitate auto-personalisation of ICT artefacts from needs and preferences (N\&P), contributing this way towards more accessible ICT for all.

In "Contrasting Usability Evaluation Methods with Blind Users" by Mei Miao, Hoai Anh Pham, Jens Friebe and Gerhard Weber, the results of two separate studies on usability methods with blind users are presented. The first study compared tactile paper prototyping and computerbased prototyping involving five blind participants. The second study compared local and synchronous remote tests with eight blind people, six visually impaired participants and six sighted persons. They conclude that in the first study, the interaction with tactile paper prototypes is very far from reality and using accessible computer-based prototypes is more intuitive for blind people, even though the of computer-based prototypes is, however, more time consuming and require good programming skills. The second study concludes that most of the blind and visually impaired participants prefer a local test instead of a synchronous remote test.

In their paper, "A Brain-Computer Interface for the Dasher Alternative Text Entry System", Thomas Welton et al. investigate whether the alternative text entry system Dasher is useful to physically and intellectually disabled students when controlled by a brain-computer interface (BCI). Seven subjects with a range of physical and intellectual disabilities participated in the study. Although Dasher-BCI performed relatively poorly compared to the use of a QWERTY keyboard, this study suggests that for people whose predominant disability is physical (cerebral palsy), BCI technology shows great potential as a viable text entry alternative.

The contribution by Daniel Zmuda, Jacek Psiuk and Marek Psiuk, entitled "Seamless Semantic Enrichment of Services in Assistive Environments", proposes a methodology and a tool-based support for the development of semantic services in ambient-assisted living (AAL)-oriented assistive environments. AAL leverages the potential of ICT to extend the period during which elderly people and people with disabilities can enjoy a healthy, safe and happy life on their own. The evaluation was performed using a sample scenario related to accessibility support. It showed that the development of semantics becomes simpler and less error prone using the proposed methodology and tool compared to existing tools.
The paper "Validation of Toolkits for developing 3rd generation Android accessible mobile applications", by Maria Gemou et al., presents the results of the evaluation of two open source toolkits that have been developed within AEGIS, aiming to support developers in the creation of accessible mobile applications, namely the Android Accessibility Designer Toolkit and the Accessibility Advisor tool. The trials were realised with 20 student developers. The results showed that $18 \%$ total development time was saved when AEGIS tools were used and that these developer tools have a big potential to help developers to create easily accessible applications.

In conclusion, this special issue presents several perspectives to provide embedded accessibility in ICT. The selected papers share a common understanding of users' requirements and the development of solutions that can fulfil their N\&P. There is an evident importance of following accessibility and personalisation standards and guidelines during the developing process of accessible solutions. These are common concerns, which the guest editor has tried to highlight with the selection of the papers included in this special issue.

The guest editor would like to thank all the reviewers for their input, as well as the authors, for achieving a great quality. Special thanks to Constantine Stephanidis, the Editor-in-Chief of the Universal Access in the Information Society international journal, for all the help during the preparation of this issue. It is hoped that readers of UAIS Journal find interesting the papers presented on this special issue.

The Guest Editor,

Maria Fernanda Cabrera-Umpiérrez.

\section{List of reviewers}

Jon Azpiroz-Vodafone, Spain

Evangelos Bekiaris-Hellenic Institute of Transport, Centre for Research and Technology Hellas, Greece

David Brown-Interactive Systems Research Group, School of Science \& Technology, Nottingham Trent University, UK

Iván Carmona Rojo-Technosite- Fundación ONCE, Spain Anton Civit-Universidad de Sevilla, Spain

Lindsay Evett-Interactive Systems Research Group, School of Science \& Technology, Nottingham Trent University, UK

Greg Fields-Research In Motion, USA

Christos Giachristis-BMT Group Limited, UK

Natalí González Villariny-Fundación Once, Spain

Joe Gorman-Stiftelsen for industriell og teknisk forskning, Norway

Peter Korn-Oracle, USA 
Jan Paul Leuteritz-Fraunhofer Institute for Industrial Engineering IAO, Germany

Clayton Lewis-University of Colorado, USA

Juan Carlos Naranjo-Instituto de Aplicaciones de las Tecnologías de la Información y de las Comunicaciones Avanzadas, Spain

Ignacio Peinado Martínez-Technosite, Spain

Jan Richards-Ontario College of Art and Design University, Canada

Alejandro Rodríguez Ascaso-Universidad Nacional de Educación a Distancia, Spain

Dario Salvi-Life Supporting Technologies, Universidad Politécnica de Madrid, Spain

Christophe Strobbe-Katholieke Universiteit Leuven, Belgium
Mari Satur Torre Calero-Fundación Vodafone, Spain

Jutta Treviranus-Ontario College of Art and Design University, Canada

Dimitrios Tzovaras-Information Technologies Institute, Centre for Research and Technology Hellas, Greece

Karel Van Isacker-European Platform for Rehabilitation, Belgium

Gregg Vanderheidem-Trace R\&D Center, University of Winsconsin-Madison, USA

Cecilia Vera Muñoz-Life Supporting Technologies, Universidad Politécnica de Madrid

Gottfried Zimmermann-Stuttgart Media University, Germany 